
\title{
Besearch S Surare \\ Intelligent Decision Making Algorithm for Balanced Cluster Formation in Wireless Sensor Networks
}

\section{Santhosh Kumar B ( $\nabla$ santhoshkumarresearch@gmail.com )}

Institute of Aeronautical Engineering

\section{Trinatha Rao P}

GITAM school of Technology, GITAM deemed to be university

\section{Research Article}

Keywords: cluster-based multiple sink, hybrid optimization algorithm, intermediate sensor, sink node and a sensor node, weighted k-mean algorithm.

Posted Date: March 9th, 2021

DOI: https://doi.org/10.21203/rs.3.rs-197461/v1

License: (1) This work is licensed under a Creative Commons Attribution 4.0 International License. Read Full License

Version of Record: A version of this preprint was published at Wireless Personal Communications on August 20th, 2021. See the published version at https://doi.org/10.1007/s11277-021-08715-6. 


\title{
Intelligent decision making algorithm for balanced cluster formation in
}

\author{
Wireless Sensor Networks \\ ${ }^{* 1}$ B. Santhosh Kumar, Dr.P. Trinatha Rao \\ *1 Department of ECE, Institute of Aeronautical Engineering, Hyderabad, Telangana, India. \\ Email Id: bsanthosh01@gmail.com \\ 2 Department of ECE, GITAM School of Technology, GITAM Deemed to be University, Hyderabad, \\ Telangana, India \\ Email Id: trinath.polipalli@gitam.edu
}

\begin{abstract}
:
In this paper, we propose an optimal cluster-based multiple sink reconnection technology for wireless sensor networks using a hybrid optimization algorithm. The proposed design used an intermediate sensor / mobile node next to the sink node and a sensor node ready to transmit data. Here, the weighted k-mean algorithm is converted to a cluster node. The optimal cluster head code was chosen using a mass optimization method that targets the available energy and available bandwidth for successfully transferring data without node optimization. So with resource updates, you can find the right location for your sink node for better data transfer.
\end{abstract}

Keywords: cluster-based multiple sink, hybrid optimization algorithm, intermediate sensor, sink node and a sensor node, weighted k-mean algorithm.

\section{Introduction:}

Wireless Sensor Network (WSN) is compatible with home network environments with features such as home architecture, infrastructure and fault tolerance. WSN has many sensors and actuators that can detect, process, and send home source data to remote source sockets. [1] [2] Typical home applications for WSN include home automation, home activity detection and home hygiene. Network sensor devices are usually small and hard to charge. Therefore, it is very important to design an energy efficient network layer algorithm to increase the home network lifetime. Recently, energy-efficient routing algorithms and protocols have been proposed to enhance WSN's network life. In this routing scenario, zinc nodes are usually fixed inside or 
outside the WSN [3] - [5]. Hotspot events inevitably occur when sensors near the sink node have a higher traffic load than the sink node on multi-hop transmissions. It consumes much faster than all available power, reduces network performance, reduces network layer, insulated nodes, and network life.

The quality of service received for these applications begins to diminish with the amount of realtime data, and most packets lose significant time. One of the solutions is to explore the gateway's ability to obtain real-time data to improve time in these situations. In this case, reinstalling the gateway balances the traffic load on multiple nodes and reduces the miss rate of real-time packets [7]. The gateway is a laptop computer or other small device that does not expect a long journey behind the rescue team. Changing the gateway during regular network operation is difficult [8] [9]. Gateway reconfiguration is where gateways go, and how gateway tasks handle data traffic. When adding real-time packets, you must first promote gateway broadcasting for traffic sharing, network performance improvements, inefficient power outages or downtime. Game Theory and Advanced Agent Colony-Based MS. Path selection and data collection (GTAC-DG) technology is used to select the best RP for MS and to evaluate the overall intelligence of advanced agent colony optimization [11]. The main objective of this study is to improve the energy consumption and network life of the sensor nodes in relation to the optimal clustering effect.

Keyword: multi-sink repositioning, queen honey bee optimization, multi-choice chaos optimization, intelligent decision making

The paper is arranged six sections. It starts with introduction in which details of the WSN, sink repositioning its application, major issues in the existing technique. The second part is the related work, in this section different papers are survived to gets idea about different technique that isinvolved. The third section is the problem definition where major problems that are involved in the current technique are mention and its possible solution. The fourth section is gives the details of proposed algorithm. The fifth section is the result and its analysis. The last section is the conclusion.

\section{Related work}


During the data transmission in the WSN the sensor nodes are closer to the sink nodes and they provide the energy earlier than nodes as they away from the data packets [15]. The advantage of WSN is energy saving and its cost is effective. Karkvandiet al. [16] introduces technique to solve the problem of linear program. AlShawiet al. [17]introduces a combination of Fuzzy approach and A-star algorithm for the improve the network lifetime. Yanget al. [18]introduces technique to reduce the energy in the node. Sensors are used to monitor and control the environment. Singhet al. [19] proposes PSO for cluster head and the data in the cluster node. Senturket al.[20]describes several factors such as battery depletion and hardware failures are the effect of wireless sensor networks. According to Thanigaivelu et.al [21] a typical wireless sensor network scenario, all the data are routed back to a static sink. Xiaet al. [22] have proposed traditional underground coalmine monitoring systems are mainly based on the use of wired transmission. Premanand and Rajaram proposed a routing scheme based on the backbone routing for the mobile ad-hoc network [27]. Rajaram and Palaniswamy proposed a trust based routing scheme in the wireless sensor network [28].

\section{Problem methodology and system model}

\subsection{Problem methodology}

In a WSN various numbers of sinks are there with large number of resources and sensors. Source is field where the data are generated. From the study it is clear that the data can be send to different multiple sinks[23]. Depending upon the real-time trafficsink relocationis used for different cases. This will reduce the life time of the node. Also due to the frequent movement of nodes the traffic of thenetwork will increase. Thus the reliability of the data transmission reduces. An optimal multi-sink repositioning (OMSR) technique is proposed using hybrid optimization algorithm. The main contributions of proposed technique are summarized as follows:

In OMSR technique, a queen honey bee optimization (QHBO) algorithm used for balanced cluster formation. It is inspired from the honey bee optimization algorithm. 
Then, compute cluster head $(\mathrm{CH})$ for each cluster using a multi-choice chaos optimization (MCO) algorithm. $\mathrm{CH}$ gathers data transmitted by its corresponding cluster member nodes and send it to base station via other $\mathrm{CHs}$.

The sink repositioning is enhanced by an intelligent decision making (IDM) algorithm under consideration of performance metrics namely distance, received signal strength, available bandwidth and cooperation rate.

\subsection{System Model}

The figure 1 shows the system model. The system consist clusters, sink and nodes. The nodes are connected to transfer data. In a cluster the cluster head is calculated. Then the data transmission is performed with respect to the cluster head. The network is divided into different cluster. For the cluster formation the used technique is the queen honey bee optimization. In the cluster different nodes arepresent. For each node the trust degrees are calculated. The trust degree values are calculated by taking the parameters like receiver signal strength(RSS), bandwidth (BW) and distance (d).By comparing the highest trust degree node is identified and that node is taken as the cluster head. For the cluster head multi choice chaos optimization algorithm is used. The data transmitted using the cluster head.Then this node is link to the sink. The sink repositioning is enhanced using the intelligent decision making algorithm.

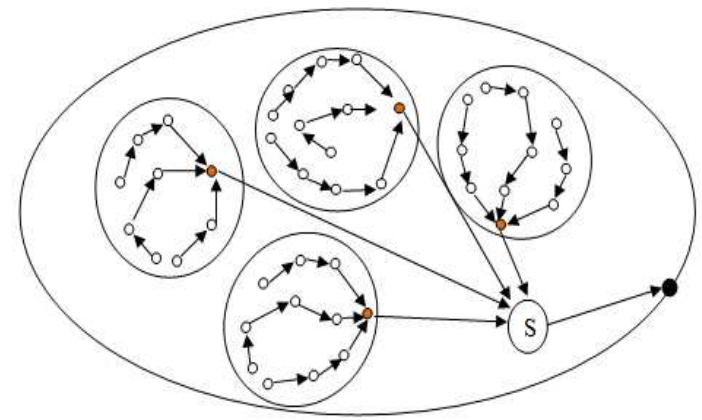

- Cluster Head

(S) $\operatorname{Sink}$

O Node

- New Location

Figure 1 System Model of proposed OMSR technique

\section{Proposed Optimal Multi-Sink Repositioning (OMSR) Technique}

\subsection{Cluster Formation using Queen Honey Bee Optimization Algorithm}


In this work, the foraging behavior of honey bees (HBAC) is used to select the CHs. Clusters are formed based on the selected CHs. The role of $\mathrm{CH}$ is assigned to other nodes which have more nectar than old one. The $\mathrm{CH}$ nodes are re-elected after all members in a cluster sent their data via a time division multiple access (TDMA) schedule. The proposed HBAC algorithm works in two phases. The first one is the cluster setup, and the second is cluster maintenance and communication phase. Node which is having different mobility and different direction is the one which is not recommended for the $\mathrm{CH}$. Thus the total energy of the node is calculated as $\mathrm{y}_{\mathrm{i}}$ :

$$
Y_{i}=E_{n}+Q_{n}+D_{n}+M_{n}
$$

For the optimization process $\mathrm{CH}$ is calculated by using the distance of each $\mathrm{CH}$. It is considered that the distance between the two $\mathrm{CH}$ is same. The distance between the food sources are approximately same. This technique will help to solve the problem of grouping that existing in the cluster. It will also reduce the overlapping of clusters. Also the position of the node is given by $\mathrm{yi}_{\mathrm{i}} \varepsilon \ln _{\mathrm{n}}$ and the average fitness value is given by $c_{j} \varepsilon l_{n}$. the weight of the node is given by $\mathrm{Z}_{\mathrm{ij}}$.

$$
\text { Minimize } \mathrm{F}(\mathrm{w}, \mathrm{c})=\sum_{i=i}^{n} \sum_{j=1}^{\mathrm{k}} z_{i j}\left(x_{i}-c_{j}\right)^{2}
$$

When $\sum_{j=1}^{k} z_{i j}=1$

$c_{j}=\frac{2}{n_{j}} \sum_{j=1}^{k} z_{i j} y_{i}$

where $n_{j}$ is the total number of node in the $j^{\text {th }}$ cluster. It is assumed that if node $i$ is assigned to a cluster $j$, the value of $\mathrm{z}_{\mathrm{ij}}$ will be 1 or 0 . This is the initialization process. The next step is the new population or new connecting clusters that are coming to the existing cluster. The clusters will accept the cluster head in the new cluster by checking the value of the $\mathrm{CH}$. The process of checking the property of the new cluster is given by the equation below. The actual quantity of nectar at node $\mathrm{i}$ is given by $\mathrm{Q}\left(\mathrm{N}_{\mathrm{i}}\right)$.

$$
\mathrm{Q}_{\mathrm{i}}=\frac{1}{1+\mathrm{cf}_{\mathrm{i}}}
$$

Where $\mathrm{cf}_{\mathrm{i}}$ is the cost function of the node and the equation of the cost function is given by:

$$
\mathrm{cf}_{\mathrm{i}}=\frac{1}{k} \sum_{i=1}^{k} d\left(x, \mathrm{~N}_{i}\right)
$$

The probability of the working bee at the node $\mathrm{y}_{\mathrm{i}}$ is given by $\mathrm{P}_{\mathrm{i}}$ :

$$
P_{i}=\frac{Q_{i}}{\sum_{k=1}^{f_{s}} F\left(\mathrm{~N}_{j}\right)}
$$


The cluster formation of the using QHB algorithm is explained in the Algorithm 1.

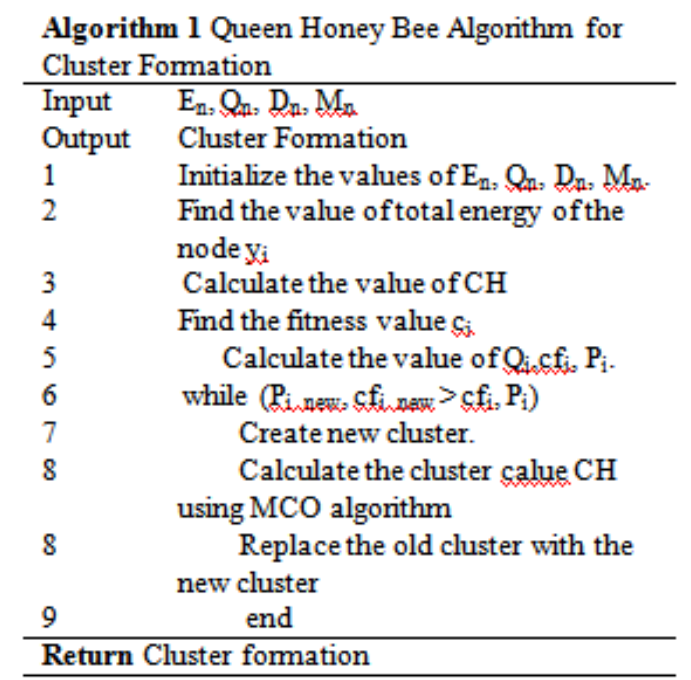

\subsection{Cluster head formation using Multi Choice Chaos Algorithm}

The $\mathrm{CH}$ is selected according to the trusted degree value. The node which is having the highest trusted value is selected as cluster head. The $\mathrm{CH}$ of one cluster can communicate with the cluster head of other cluster. Received signal strength (RSS) is measured by taking the distance and transmission energy. The equation of RSS is given by:

$$
R S S=\frac{F F E(n, d)}{4 \pi D_{i}^{2}}+T_{a, a_{1} / a_{2}}
$$

The life time is the maximum time duration of network and the perfect network maintains congestion should be less and high life time.

The distances (d) between the clusters are calculated using Euclidian distance and this distance is same for the entire cluster head.

$$
d=\sqrt{\left(x_{A}-x_{B}\right)^{2}+\left(y_{A}+y_{B}\right)^{2}}
$$

where $\left(\mathrm{x}_{\mathrm{A}}, \mathrm{y}_{\mathrm{B}}\right)$ is the position of node $\mathrm{A}$ in the network and $\left(\mathrm{x}_{\mathrm{B}}, \mathrm{y}_{\mathrm{B}}\right)$ is the position of node $\mathrm{B}$ in the network. Consequently, Euclid distance between two nodes has a square relation with the sent energy. The equation for calculating the BW is given by:

$$
\mathrm{BW}=\frac{\mathrm{Ns}(\mathrm{CH}(\mathrm{n})+1)}{1}
$$

Where Ns all sensing child nodes, 1 is the length of the length of the link. If the BW is highthen the QoS will high it will improve the performance of the network.

Depending on all the above mention condition the trust values are calculated for every node. Then, the depending on those values the $\mathrm{CHs}$ are assigned for each cluster. The trust values for neighbor nodes are given by $\mathrm{T}_{\mathrm{n}}$ :

$$
\mathrm{T}_{\mathrm{n}}=\operatorname{Avg}(\mathrm{RSS}, \mathrm{BW}, \mathrm{d})
$$




\subsubsection{Multi-Choice Chaos Optimization (MCO) Algorithm}

The 'chaos' means something that cannot be predicted.Chaotic phenomena are involved when chaos is combined with the deterministic.Chaotic phenomena involve specific laws, mathematical apparatus and a physical origin. There are no random or any stochastic effects in the deterministic chaos. A discrete chaotic map is a type of deterministic chaos. It uses iterated functions to determine the function. For the $\mathrm{CH}$ formation the used map is the Lozi map, the common equation is given by:

$$
\begin{gathered}
X_{n+1}=1-a\left|X_{n}\right|+b Y_{n} \\
Y_{n+1}=X_{n}
\end{gathered}
$$

This is used because it is simple type map that are used for repeated comparison of the values in the group of areas.The cluster head formation of the using MCO algorithm is explained in the Algorithm 2.

\section{Sink Repositioning usingIntelligent Decision Making}

From biological species like shoals of fish, flocks of birds, and colonies of ants IDM is originated. It is commonly used for analyzing the unsophisticated agent interaction locally with their environment. This interaction will make coherent functional global patterns.It consists of s particlesand it is operating in $\mathrm{n}$ dimensional space. The number of nodes to be optimized is given by $\mathrm{n}$. The position of the node is given by $\mathrm{Xi}_{\mathrm{i}}$ and the velocity of the node is given by $\mathrm{c}_{\mathrm{i}}$. The value of $\mathrm{i}$ is given by $1 \leq i \leq s$. Initially the position of the node is given by $\mathrm{x}_{\min }<\mathrm{xi}_{\mathrm{i}}<\mathrm{x}_{\max }$ and $\mathrm{c}_{\min }<\mathrm{c}_{\mathrm{i}}<\mathrm{c}_{\max }$.

$$
\begin{aligned}
& \text { Algorithm } \\
& \text { Input: RSS, BW, d, Tno } \\
& \text { Output: } \mathrm{CH} \\
& 1 \text { Initialize network } \\
& 2 \text { For each node the trust value }\left(T_{n}\right) \text { is } \\
& \text { calculated. } \\
& 3 \text { Send the node value to the neighbor node } \\
& 4 \text { while( }\left(T_{n}\right. \text { of clusters are compare) } \\
& 5 \text { Cluster havinghighest } T_{n} \text { is calculated as } \\
& \mathrm{CH} \text {. } \\
& \text { For all cluster the } \mathrm{CH} \text { value is } \\
& \text { calculated } \\
& 7 \text { end } \\
& \text { Return } \mathrm{CH} \text { value } \\
& c_{i}(k+1)=w \cdot c_{i}(k)+c_{1} \cdot \operatorname{rand}_{1} \cdot\left(p_{\text {best }}-x_{i}\right)+c_{2} \operatorname{rand}_{2} \cdot\left(g_{\text {bes }} t-x_{i}\right) \\
& x_{i}(k+1)=x_{i}(k)+c_{1} \cdot(k+1)
\end{aligned}
$$

where rand $\mathrm{d}_{1}$ and rand $_{2}$ are random numbers having uniform distribution in the range $(0,1), \mathrm{w}$ is the inertia coefficient and its value various from $0.2<\mathrm{w}<1.2$, the velocity is given to each node and it is given by $\mathrm{X}_{\min }$ and $\mathrm{x}_{\max }$. Magnitude and direction of movement of a particle is influenced by its previous velocity, its experience and the knowledge it acquires from the swarm through social interaction. The working function of sink repositioning using the IDM algorithm is given in Algorithm 3. 


\section{Result and Discussion}

The simulations are performed using NS2 simulation environment. The simulations are carried out with metrics for both proposed systems and existing. From the comparison it is clear that existing LTRCORC technique [25] has low performance as compared to the proposed system OMSR. For the simulation process the total number nodes varied from 10000 to 90000 . The operating bandwidth is $2.4 \mathrm{GHz}$. The initial energy of the node is given by $18520 \mathrm{~J}$. The network area is considered as $500 \times 500$ $\mathrm{m}$. It is taken that the topology is a uniform type. The table 1 shows the parameter values that are used for the simulation.

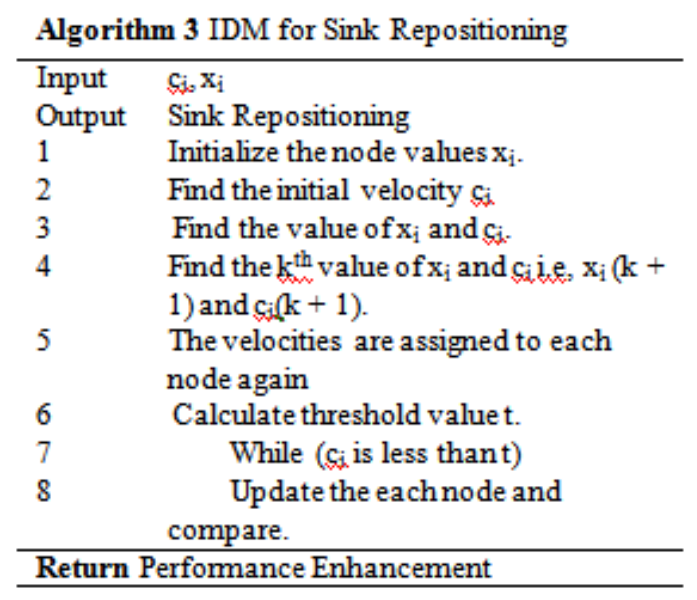

Table 1: Performance Parameters

\begin{tabular}{ll}
\multicolumn{1}{c}{ Parameter } & \multicolumn{1}{c}{ Value } \\
\hline No: of node & $10000-90000$ \\
Velocity of node & $1 \mathrm{~m} / \mathrm{s}$ \\
Simulation area & $100 \mathrm{~m}$ \\
Initial energy & $18,520 \mathrm{~J}$ \\
Topology & Uniform \\
Area & $500 \times 500 \mathrm{~m}$ \\
Threshold voltage & $100 \mathrm{mv}$ \\
Data rate & $250 \mathrm{kbps}$ \\
BW & $2.4 \mathrm{GHz}$ \\
Packet size & $97 \mathrm{bytes}$ \\
\hline
\end{tabular}

\subsection{Varying number of nodes}


This section simulation is carried out with the number of node as the common parameter. The simulation graph of the security level is shown in the figure 2. It is clear that the proposed OMSR technique is having high security as compared to the existing system. From the graph there is $10 \%$ improvement in the security level when OMSR is used. The next parameter is the energy consumption. The simulation graph of energy consumption isshown in the figure 3. It is clear that the OMRS technique will reduce the energy by $27 \%$ as compared to the existing LTRC-ORC technique.The figure 4 shows the simulation analysis of the false. There false positive rate for the $45 \%$ less than the existing LTRC-ORC technique. The figure 5shows simulation result of the packet delivery ratio. From the analysis it is clear that the delivery ratio of the proposed OMSR technique has an improvement of $20 \%$. The figure 6 shows the simulation result of the network life time. It is clear that by using the proposed technique the lifetime of the network increases by $27.5 \%$. The figure7 shows the simulation graph of the packet loss. The graph shows that packet loss is low for the proposed technique.

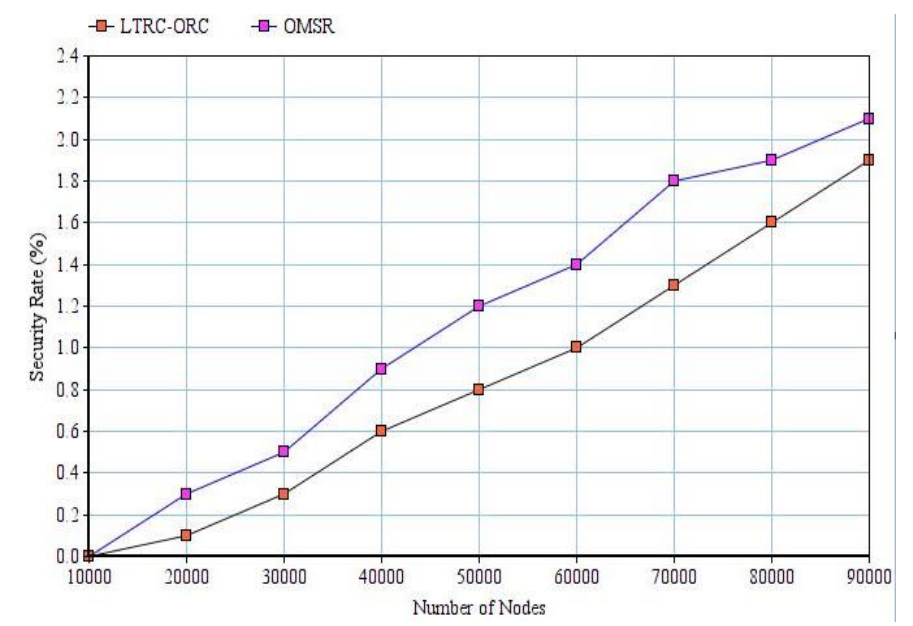

Figure 2 Security Level with Number of Node

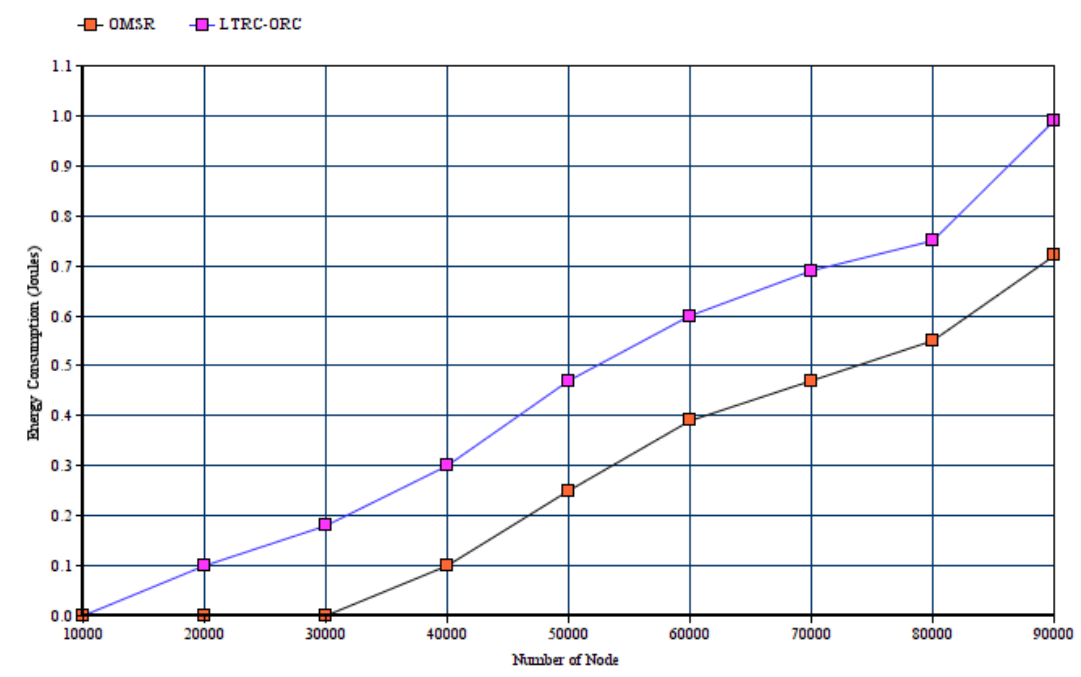

Figure 3 Energy Consumption with Number of Node 


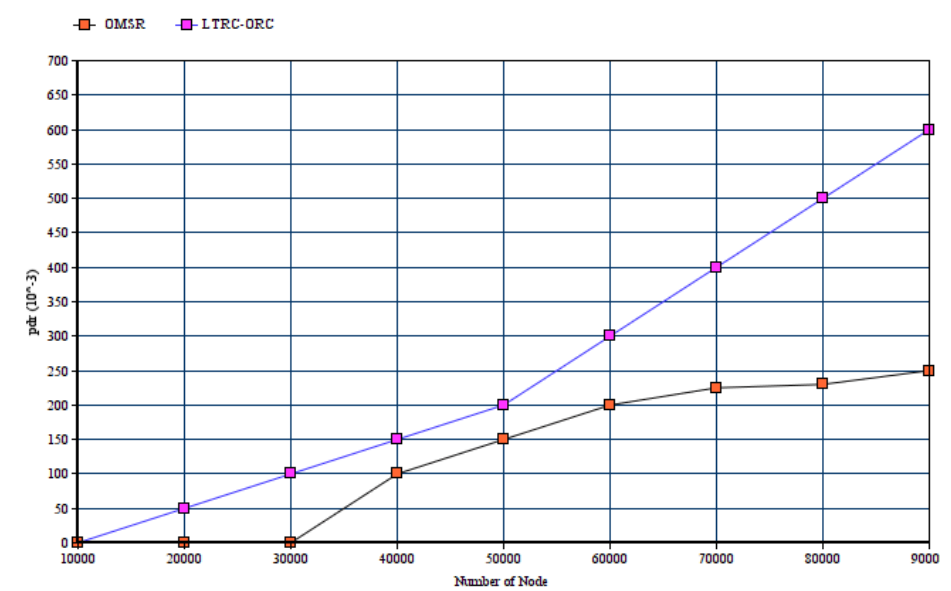

Figure 4False Position Rate with Number of Node - $\mathrm{T}$ - TRC.ORC $\square$ OMSR

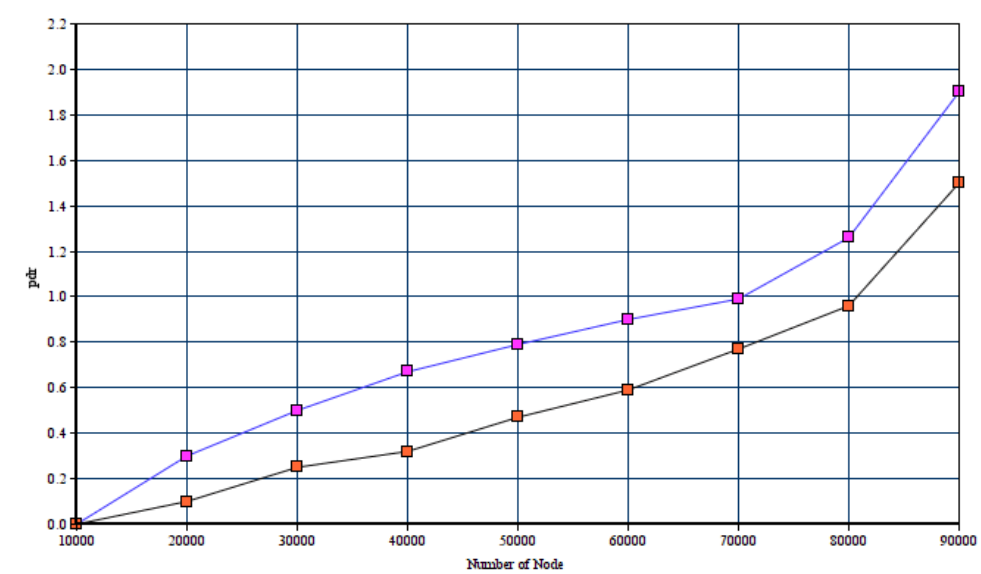

Figure 5 Packet Delivery Rate with Number of Node ㄴ. OMSR ㄴ. ITRC-ORC

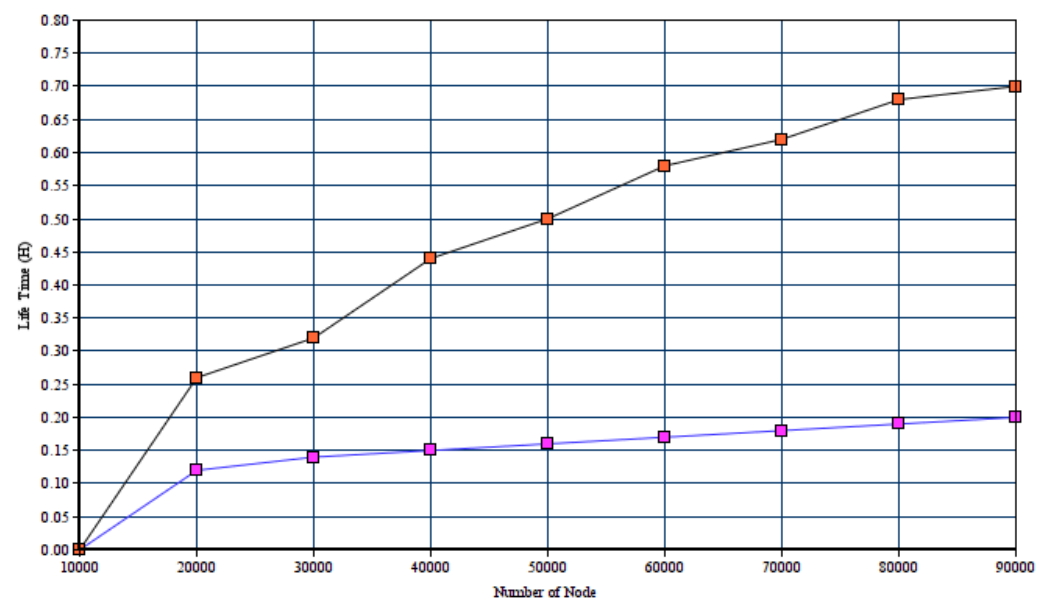

Figure 6Network Lifetime with Number of Node 


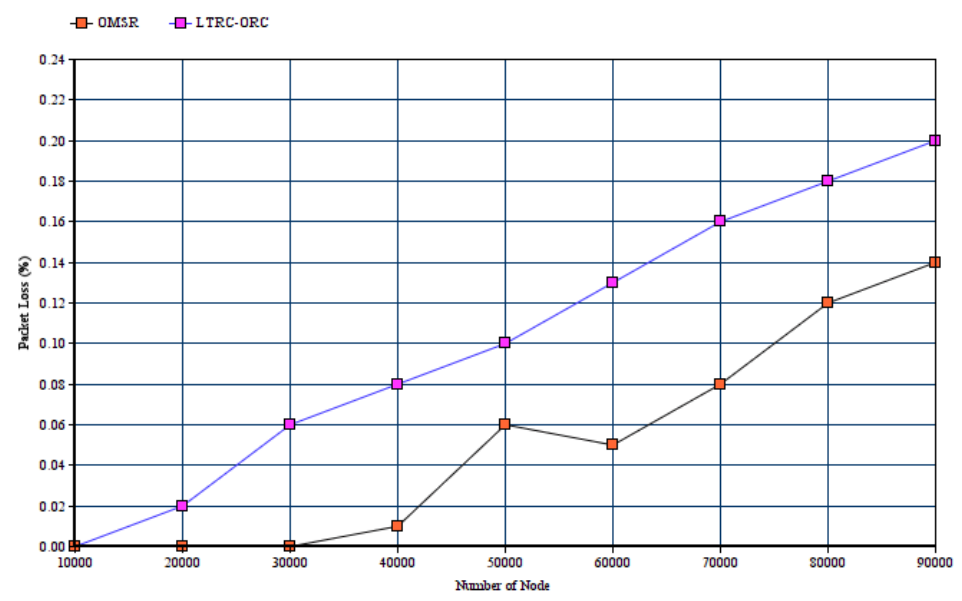

Figure 7 Packet Losswith Number of Node

\subsection{Varying the Mobility}

As similar to that of 5.1 all the parameters are simulated with the constant parameter mobility. The variation of theanalysis is shown in the below. The figure 8 shows the simulation analysis of the security level. From the analysis it is clear that there is a $30 \%$ of improvement of security level in the proposed OMSR technique.The figure 9 shows the simulation analysis of the energy consumption. From the analysis it clear that the proposed technique OMSR reduces the energy consumption by54\%. The simulation analysis of the false positive rate is given in the figure 10 . The graph shows that the using OMSR technique it is possible to identify false $54 \%$ as compared to the LTRC-ORC. Figure 11 shows the simulation of the packet delivery ratio. OMSR has an improvement of $46 \%$. Network lifetime simulation analysis is given in the figure 12 . The network lifetimeincreased by $55 \%$ when OMSR technique is used. Simulation of the packet loss is given in the figure 13. The packet loss is reduced by a percentage of $50 \%$.

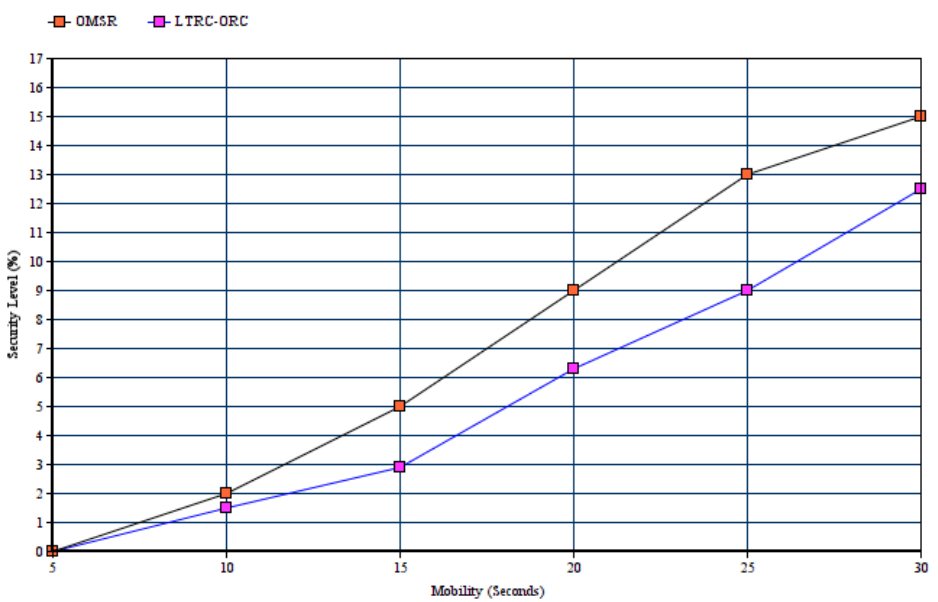

Figure 8 Security level with Mobility 


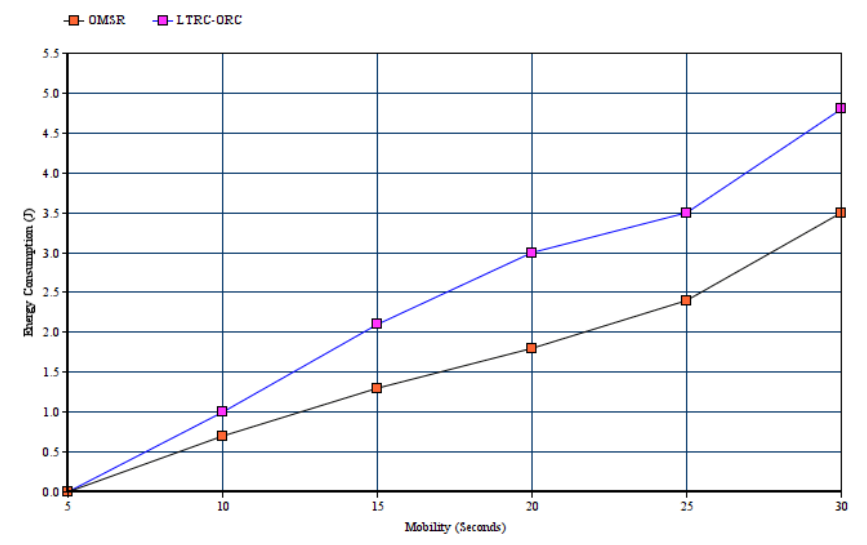

Figure 9 Energy Consumption with Mobility

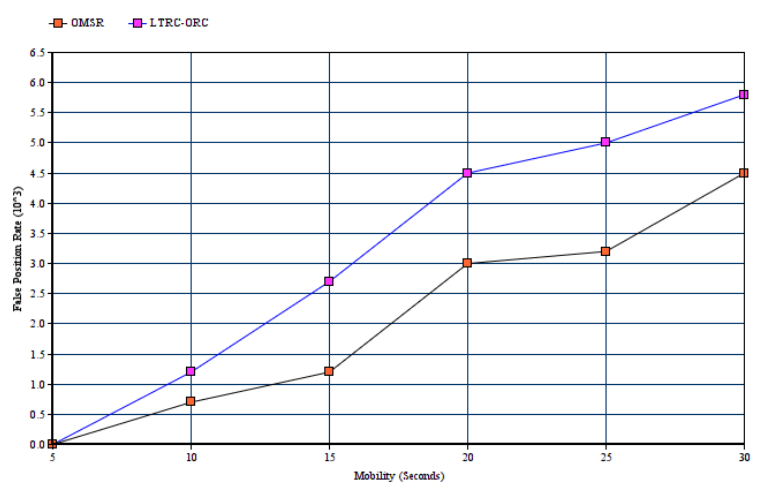

Figure 10 False Position Rate with Mobility

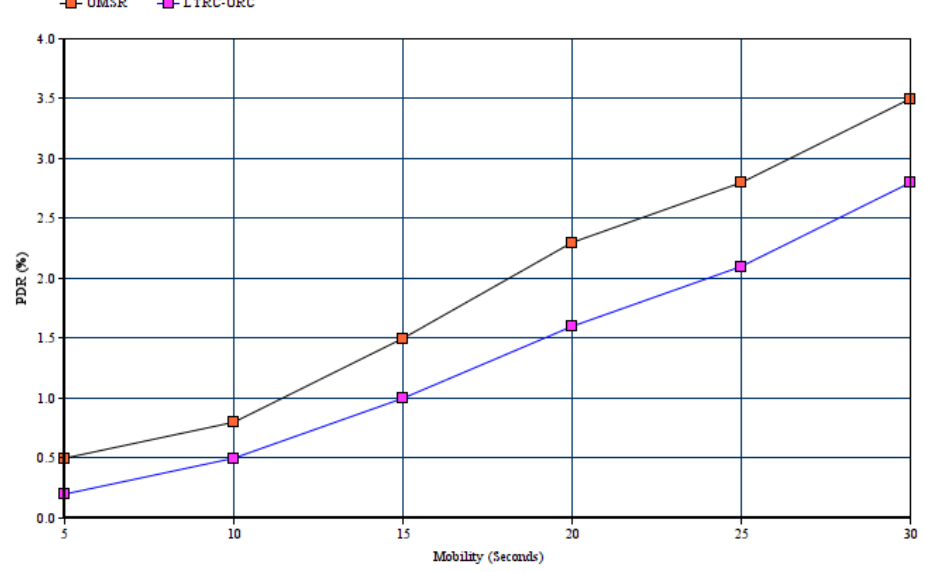

Figure 11 Packet Delivery Ration with Mobility 


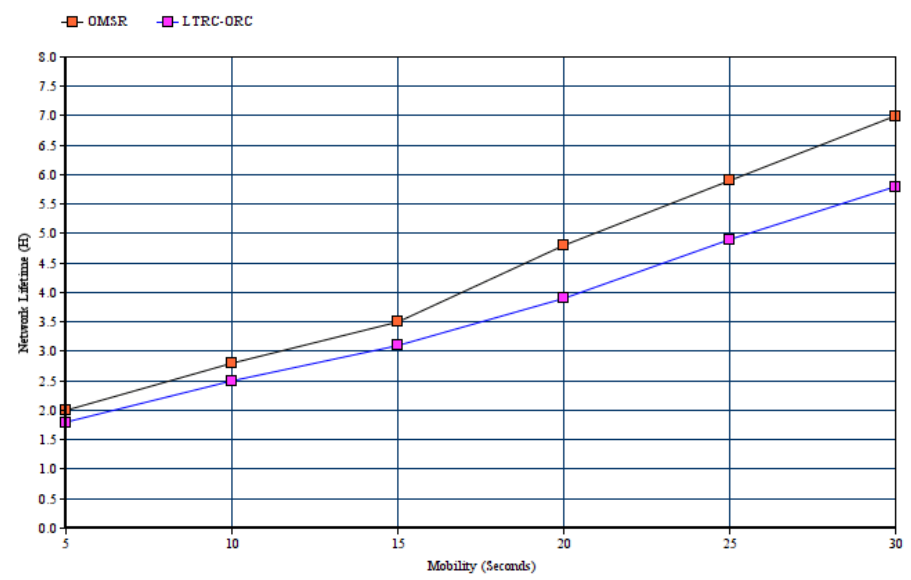

Figure 12 Network Lifetime with Mobility

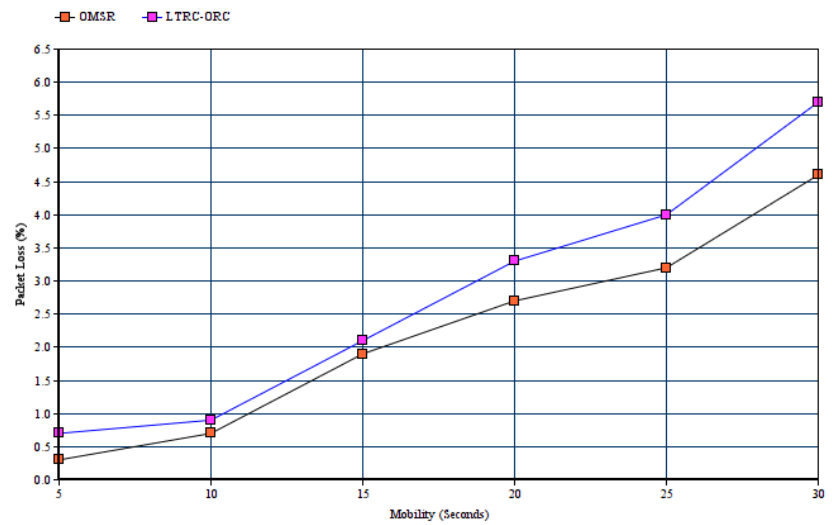

Figure 13 Packet Loss with Mobility

\section{Conclusion}

In a WSN there are sets of resource constrained sensor nodes. These nodes are normally used for monitoring and transmitting the data of the particular region. Thus the two main parameters that are to be considered are the network life time and reliable transmission of the data. Thus the sink relocation is the best method for optimizing these issues in the WSN. By comparing different technique a new algorithm is proposed and is named as OMSR. In the proposed algorithm first the clusters are formed using the algorithm QHBO. After that the cluster head values are calculated using the algorithm MCO. Finally for the enhanced of the sink repositioning IDM is used. The simulation is carried out in NS2.Security level, energy consumption, packet delivery ration, false positive rate, network lifetime, packet are the parameters that are simulated in the NS2. From the analysis it is clear that the proposed algorithm is having high performances as compared to the existing algorithm.

\section{Declarations}

\section{Funding}

No Funding involved.

\section{Conflict of Interest}

There is no conflict of interest

\section{Availability of data and Material}

Not available in websites.

\section{Author's contributions}


The first author formatted the paper and implementation under the guidance of the second author.

\section{Reference}

[1] Akkaya, K., Younis, M. and Bangad, M., 2005. Sink repositioning for enhanced performance in wireless sensor networks. Computer Networks, 49(4), pp.512-534.

[2] Khan, M.A., Hasbullah, H. and Nazir, B., 2013. Multi-node repositioning technique for mobile sensor network. AASRI Procedia, 5, pp.85-91.

[3] Ray, A. and De, D., 2016. An energy efficient sensor movement approach using multi-parameter reverse glowworm swarm optimization algorithm in mobile wireless sensor network. Simulation modelling practice and theory, 62, pp.117-136.

[4] Basagni, S., Carosi, A., Petrioli, C. and Phillips, C.A., 2011. Coordinated and controlled mobility of multiple sinks for maximizing the lifetime of wireless sensor networks. Wireless Networks, 17(3), pp.759-778.

[5] Srivastava, J.R. and Sudarshan, T.S.B., 2015. Energy-efficient cache node placement using genetic algorithm in wireless sensor networks. Soft Computing, 19(11), pp.3145-3158.

[6] Tamandani, Y.K., Bokhari, M.U. and Kord, M.Z., 2017. Computing geometric median to locate the sink node with the aim of extending the lifetime of wireless sensor networks. Egyptian Informatics Journal, 18(1), pp.21-27.

[7] Chanak, P., Banerjee, I. and Sherratt, R.S., 2017. Energy-aware distributed routing algorithm to tolerate network failure in wireless sensor networks. Ad Hoc Networks, 56, pp.158-172.

[8] Silvestri, S. and Goss, K., 2017. MobiBar: An autonomous deployment algorithm for barrier coverage with mobile sensors. Ad Hoc Networks, 54, pp.111-129.

[9] Vatankhah, A. and Babaie, S., 2018. An optimized bidding-based coverage improvement algorithm for hybrid wireless sensor networks. Computers \& Electrical Engineering, 65, pp.1-17.

[10] Narendran, M. and Prakasam, P., 2017. An energy aware competition based clustering for cluster head selection in wireless sensor network with mobility. Cluster Computing, pp.1-10.

[11]Raj, P.P., Khedr, A.M. and Al Aghbari, Z., 2020. Data gathering via mobile sink in WSNs using game theory and enhanced ant colony optimization. Wireless Networks, pp.1-16.

[12] C.-g. Tan, K. Xu, J.-X. Wang, and S.-q. Chen, "A sink moving scheme based on local residual energy of nodes in wireless sensor networks," Journal of Central South University of Technology, vol. 16, pp. 265-268, 2009. 
[13] L. B. Saad and B. Tourancheau, "Towards an efficient positioning of mobile sinks in wireless sensor networks inside buildings," in New Technologies, Mobility and Security (NTMS), 2009 3rd International Conference on, 2009, pp. 1-5.

[14] S. Basagni, A. Carosi, C. Petrioli, and C. A. Phillips, "Coordinated and controlled mobility of multiple sinks for maximizing the lifetime of wireless sensor networks," Wireless Networks, vol. 17, pp. 759-778, 2011.

[15] A. K. Singh, N. Purohit, and S. Varma, "Analysis of lifetime of wireless sensor network with base station moving on different paths," International Journal of Electronics, vol. 101, pp. 605-620, 2014.

[16] H. R. Karkvandi, E. Pecht, and O. Yadid-Pecht, "Effective lifetime-aware routing in wireless sensor networks," IEEE Sensors Journal, vol. 11, pp. 3359-3367, 2011.

[17] I. S. AlShawi, L. Yan, W. Pan, and B. Luo, "Lifetime enhancement in wireless sensor networks using fuzzy approach and A-star algorithm," 2012.

[18] Y. Yang, M. I. Fonoage, and M. Cardei, "Improving network lifetime with mobile wireless sensor networks," Computer Communications, vol. 33, pp. 409-419, 2010.

[19] B. Singh and D. K. Lobiyal, "A novel energy-aware cluster head selection based on particle swarm optimization for wireless sensor networks," Human-Centric Computing and Information Sciences, vol. 2, p. 13, 2012.

[20] I. F. Senturk, K. Akkaya, and S. Yilmaz, "Relay placement for restoring connectivity in partitioned wireless sensor networks under limited information," Ad hoc networks, vol. 13, pp. 487-503, 2014.

[21] K. Thanigaivelu and K. Murugan, "Grid-based clustering with predefined path mobility for mobile sink data collection to extend network lifetime in wireless sensor networks," IETE Technical Review, vol. 29, pp. 133-147, 2012.

[22] X. Xia, Z. Chen, H. Liu, H. Wang, and F. Zeng, "A routing protocol for multisink wireless sensor networks in underground coalmine tunnels," Sensors, vol. 16, p. 2032, 2016.

[23] X. Li, A. Nayak, and I. Stojmenovic, "Sink mobility in wireless sensor networks," Wireless Sensor and Actuator Networks: Algorithms and Protocols for Scalable Coordination and Data Communication, pp. 153-184, 2010. 
[24] M. Ahmad, A. A. Ikram, R. Lela, I. Wahid, and R. Ulla, "Honey bee algorithm-based efficient cluster formation and optimization scheme in mobile ad hoc networks," International Journal of Distributed Sensor Networks, vol. 13, p. 1550147717716815, 2017.

[25] A. Pushpalatha and G. Kousalya, "A prolonged network life time and reliable data transmission aware optimal sink relocation mechanism," Cluster Computing, pp. 1-10, 2018.

[26] Kumar, B. and Rao, P., 2020. Trusted Cluster based Optimal Multi-sink Repositioning Technique for WSNs using Improved Bull Optimization and Clonal Selection Algorithm. Test engineering and management, 2020/2

[27] Premanand, R. P., \& Rajaram, A. (2020). Enhanced data accuracy based PATH discovery using backing route selection algorithm in MANET. Peer-to-Peer Networking and Applications, 13(6), 20892098.

[28] Rajaram, A., \& Palaniswami, D. S. (2009). A trust based cross layer security protocol for mobile ad hoc networks. arXiv preprint arXiv:0911.0503.

\section{Author details}

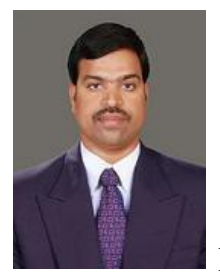

B.Santhosh Kumar ( Beledha Santhosh Kumar) is an Assistant Professor in the Department of Electronics and Communication Engineering, Institute of Aeronautical Engineering, Hyderabad, India. He is currently pursuing Ph.D in Wireless Sensor Networks from GITAM University, Hyderabad, India. He has completed his Master of Technology in Embedded systems, Joginpally B.R. Engineering College, Hyderabad, India. He Completed his Bachelor of Technology in Electronics and Communication Engineering from Madhira Institute of Technology \& Science, Kodad, Nalgonda, India. He has more than 10 years of Teaching Experience. He has published more than 10 research papers in International Journals and Conferences.

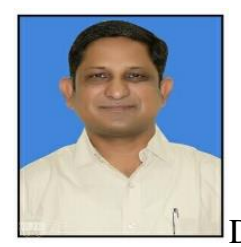

Dr. P. Trinatha Rao ( Trinatha Rao Polipalli ) is a Professor in the Department of Electronics and Communication Engineering, GITAM School of Technology, GITAM Deemed to be University, Hyderabad, India. He did his Ph.D in Communication Networks from College of Engineering, Andhra University, Visakhapatnam, India. He has completed his Masters in Engineering with majors in Optical Communication, College of Engineering, Guindy, Chennai, India. He Completed his Bachelor of Engineering in Electronics and Communication Engineering from College of Engineering, GITAM, Visakhapatnam, India. He has more than 16 years of Teaching and Research Experience. He is presently guiding 13 research scholars in the areas of Cognitive Radio, Software defined Networks etc. He has published more than 42 research papers in International Journals and Conferences. He is 
presently the Professor In charge for the Student Affairs, GITAM University, Hyderabad.

$\mathrm{He}$ is the Editorial Board member for different Journals. He was a Key note Speaker in many University and Government Organizations. He was also chair for the different Conferences and Seminars. He has reviewed books in the area of Optical Fiber Communications. One of the research paper titled, "Routing Protocols in Wireless Sensor Networks: A Survey" has been awarded as Best Research Paper by a renowned Journals. He was honoured with BEST RESEARCHER AWARD -2017, received from Honourable Vice-President of India, Sri Venkaiah Naidu, Sri T. Harish Rao, Minister for Irrigation, Marketing \& Legislative Affairs (Government of Telangana), 18 November, 2017. 
Figures
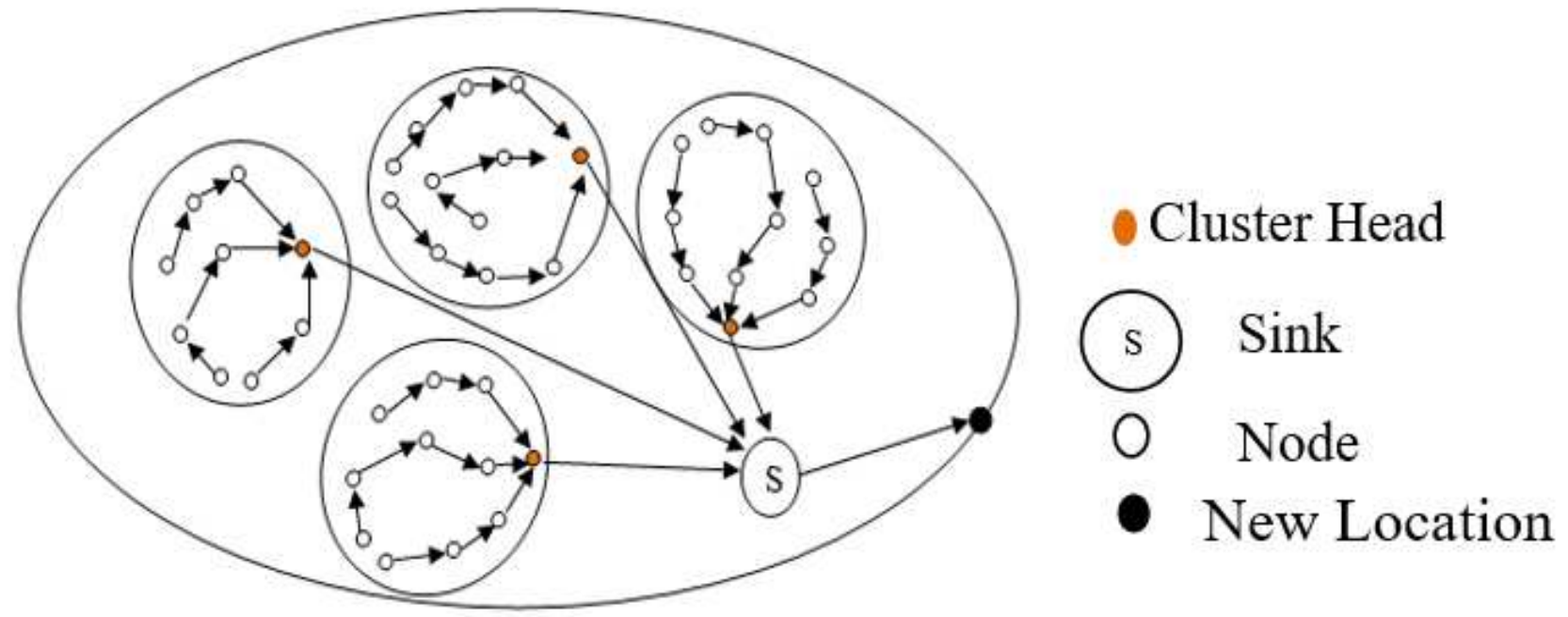

Figure 1

System Model of proposed OMSR technique

$\rightarrow$ LTRC-ORC $\rightarrow$ OMSR

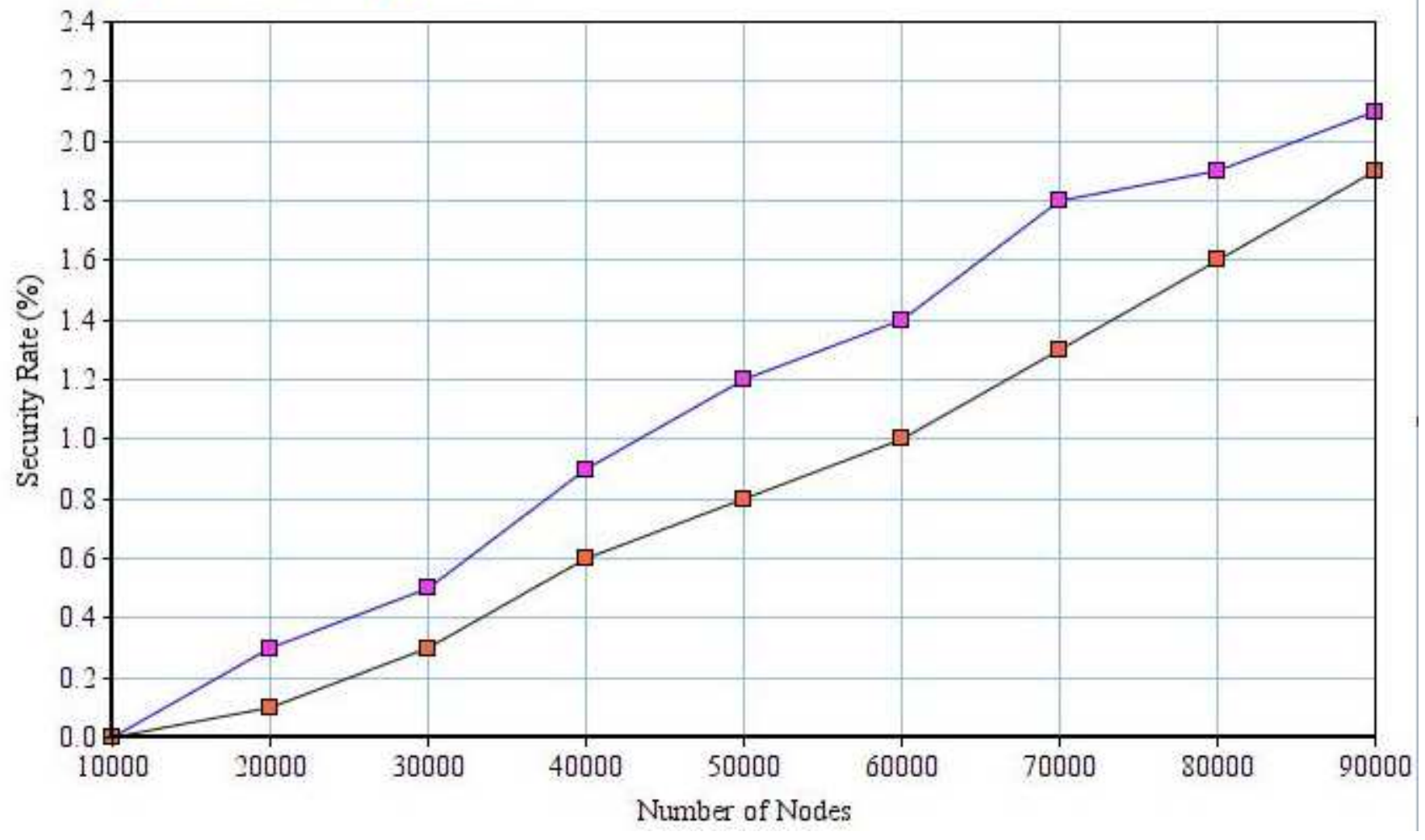

Figure 2 
Security Level with Number of Node

$\neg$ - OMSR

$\square$ L TRC-ORC

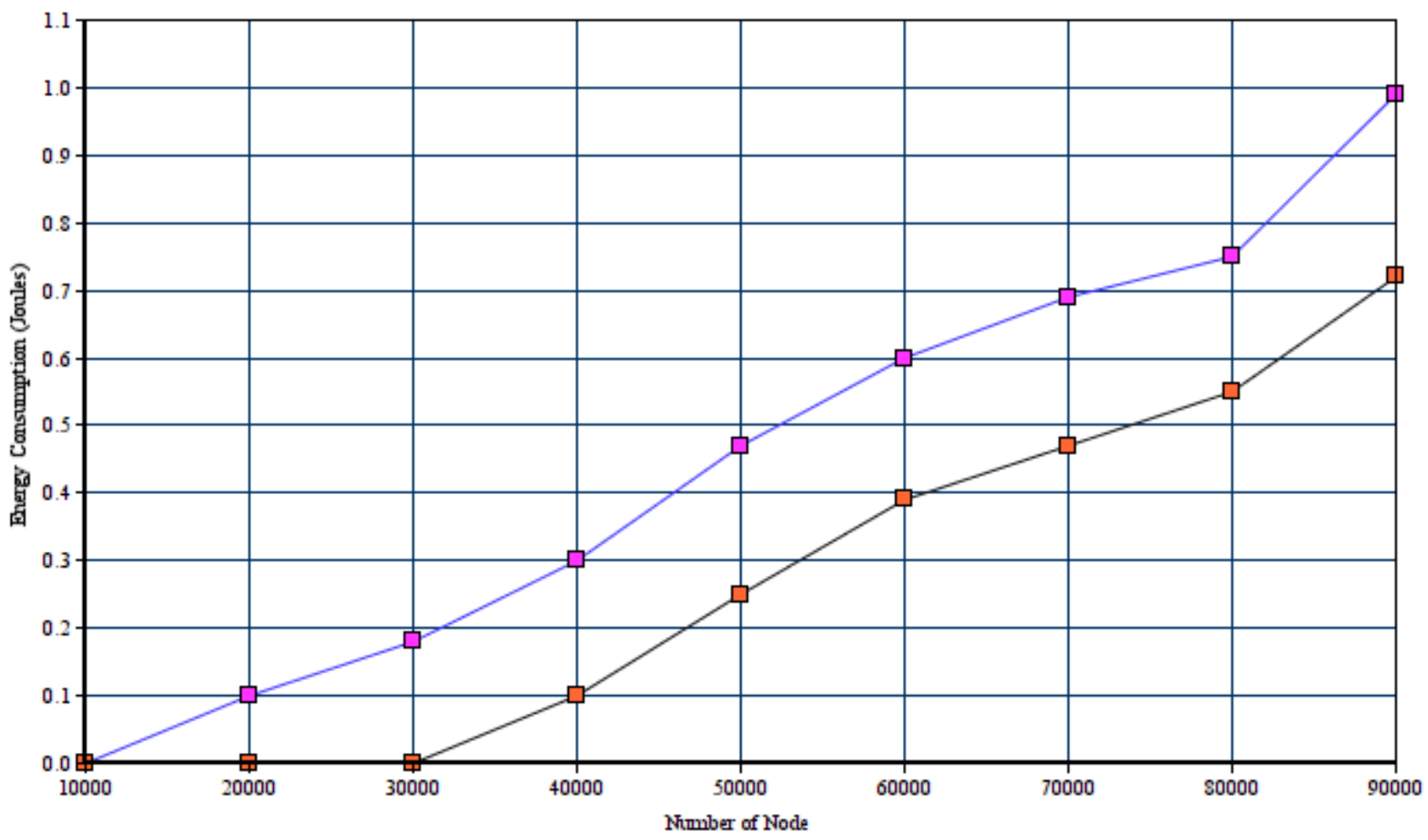

Figure 3

Energy Consumption with Number of Node

$\neg$ OMSR $\square$ - LTRC-ORC

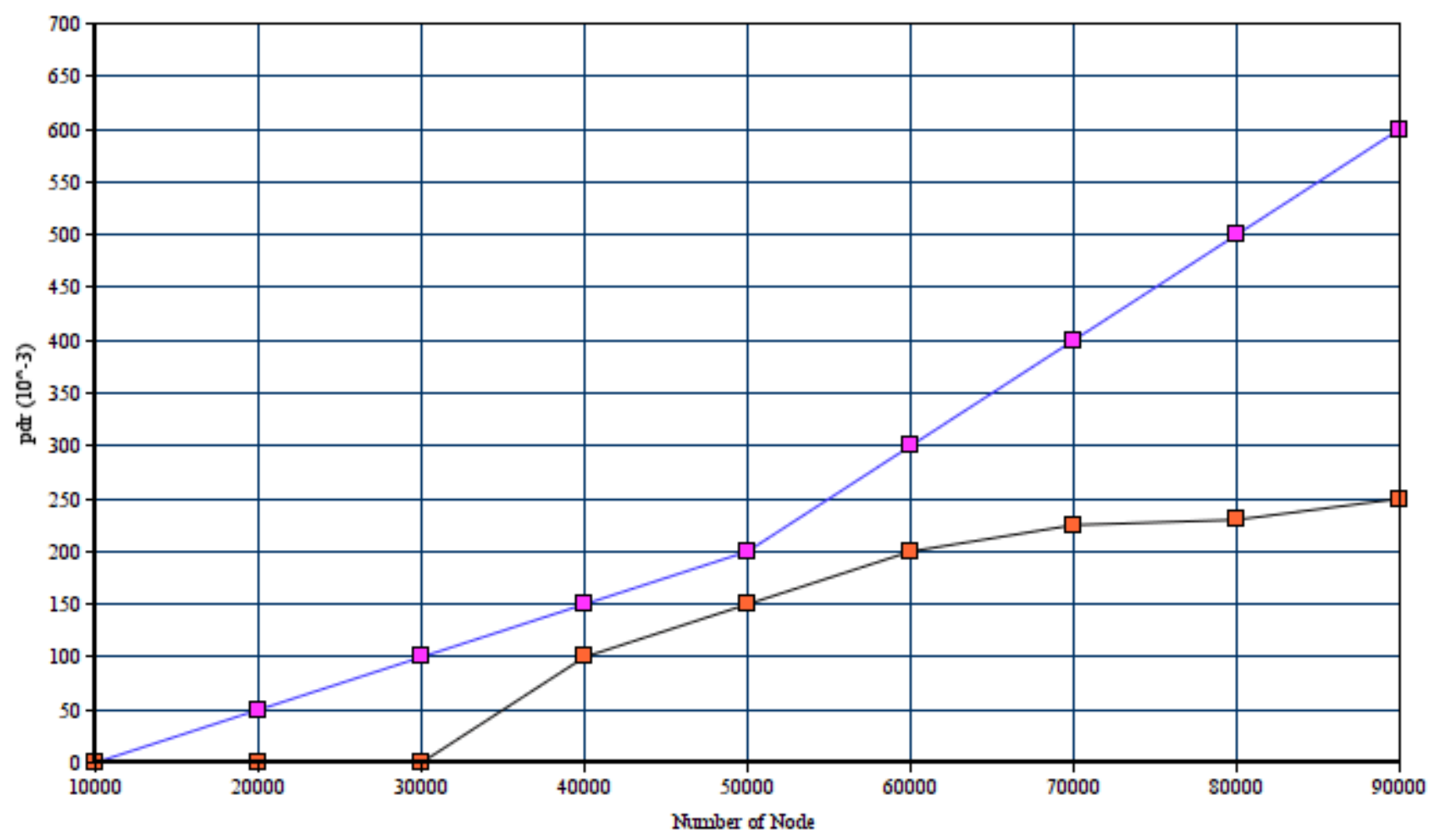


Figure 4

False Position Rate with Number of Node

$\neg$ - ITRC-ORC $\square$-OMSR

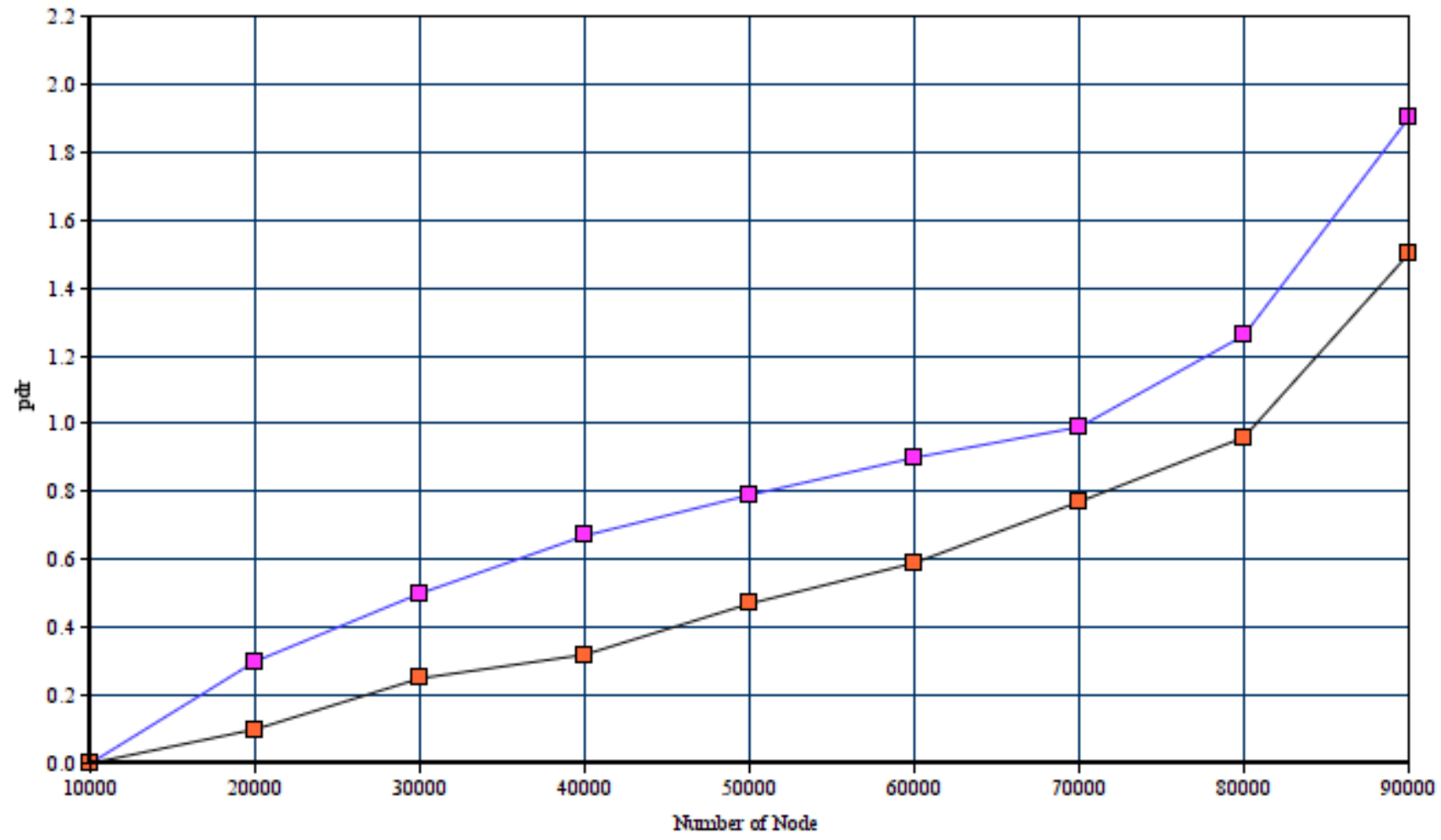

Figure 5

Packet Delivery Rate with Number of Node 


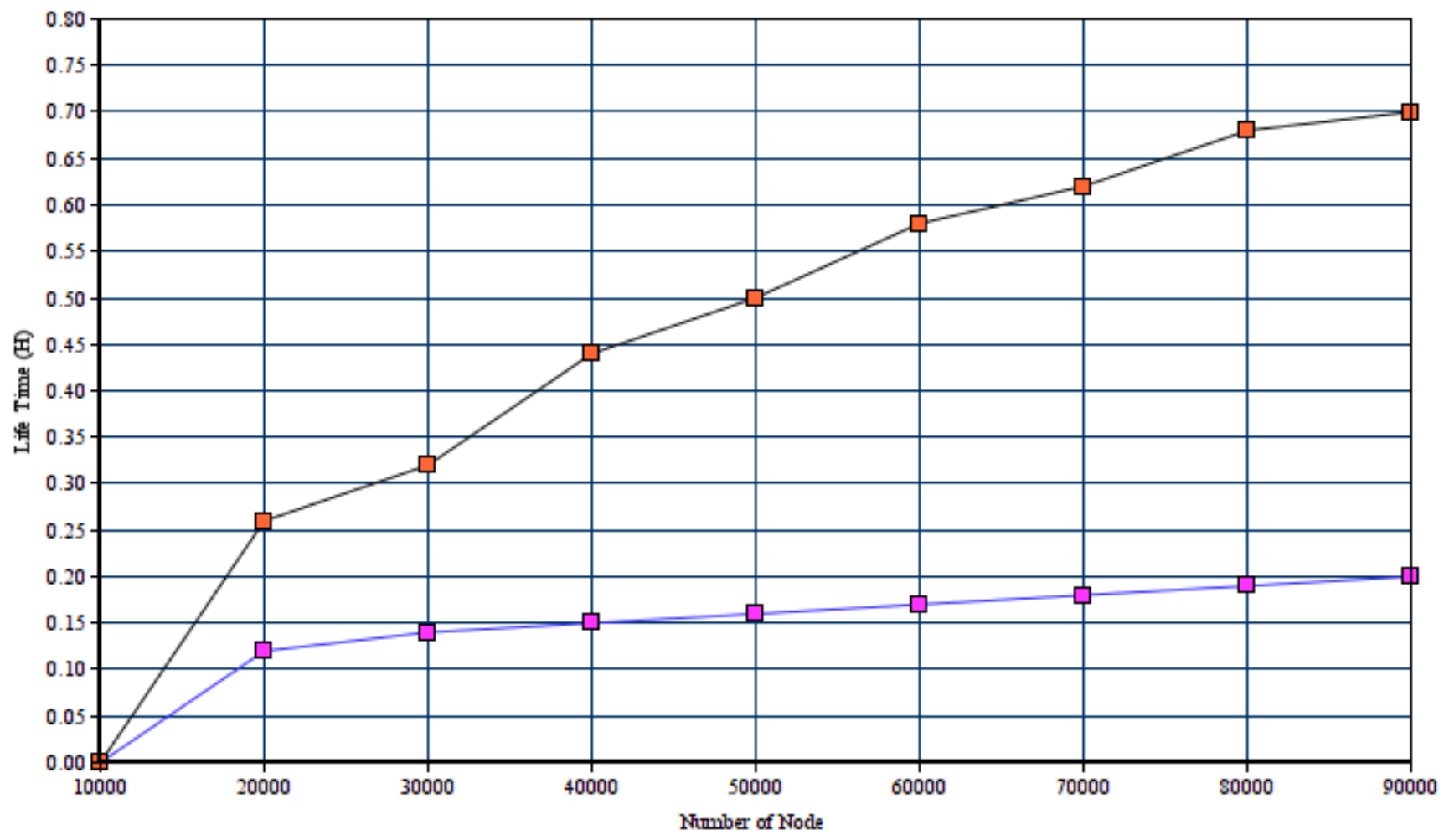

Figure 6

Network Lifetime with Number of Node

- $\square$ OMSR $\square$ LTRC-ORC

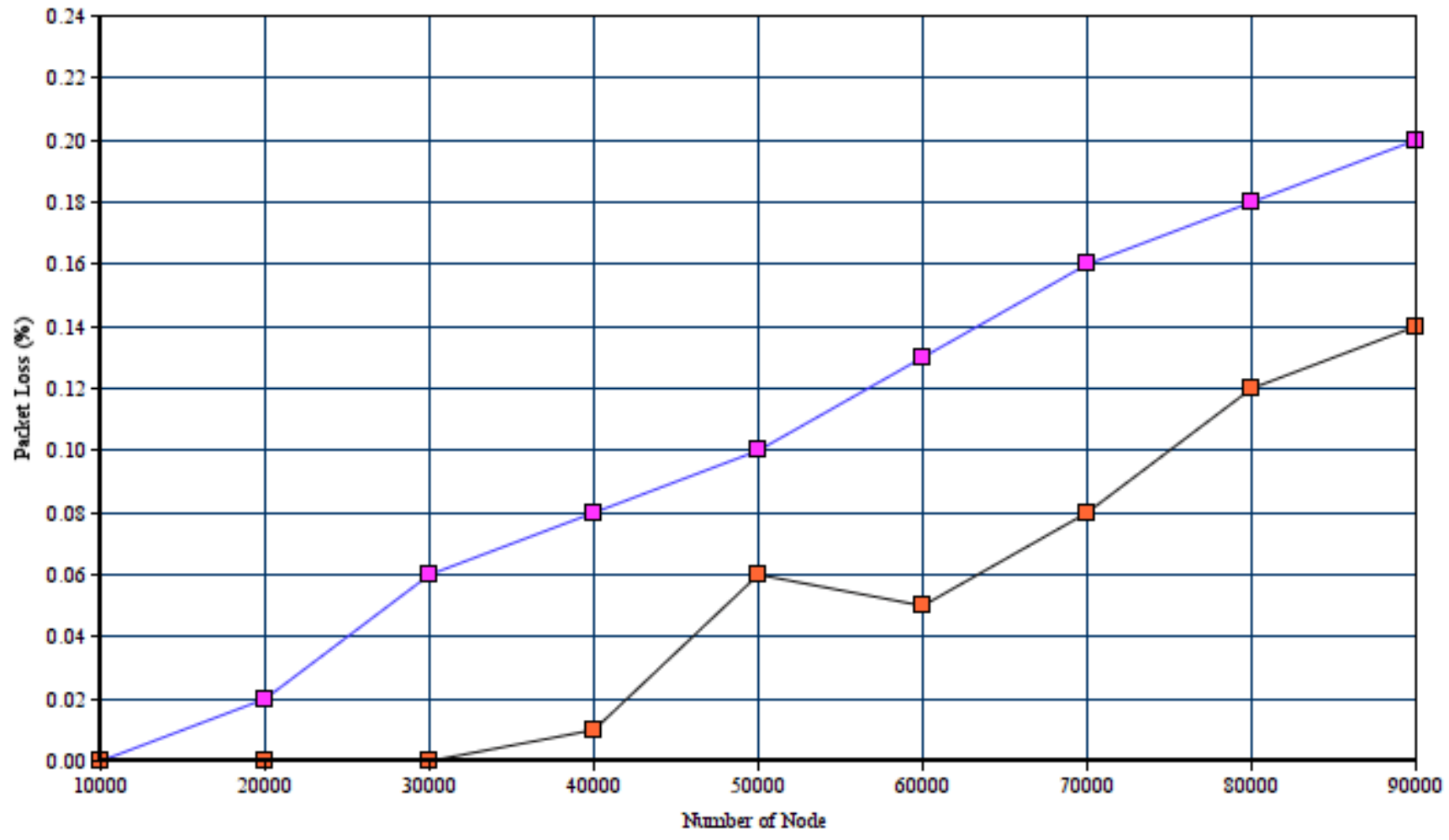

Figure 7 
Packet Loss with Number of Node

$\neg \square$ OMsR

- - L TRC-ORC

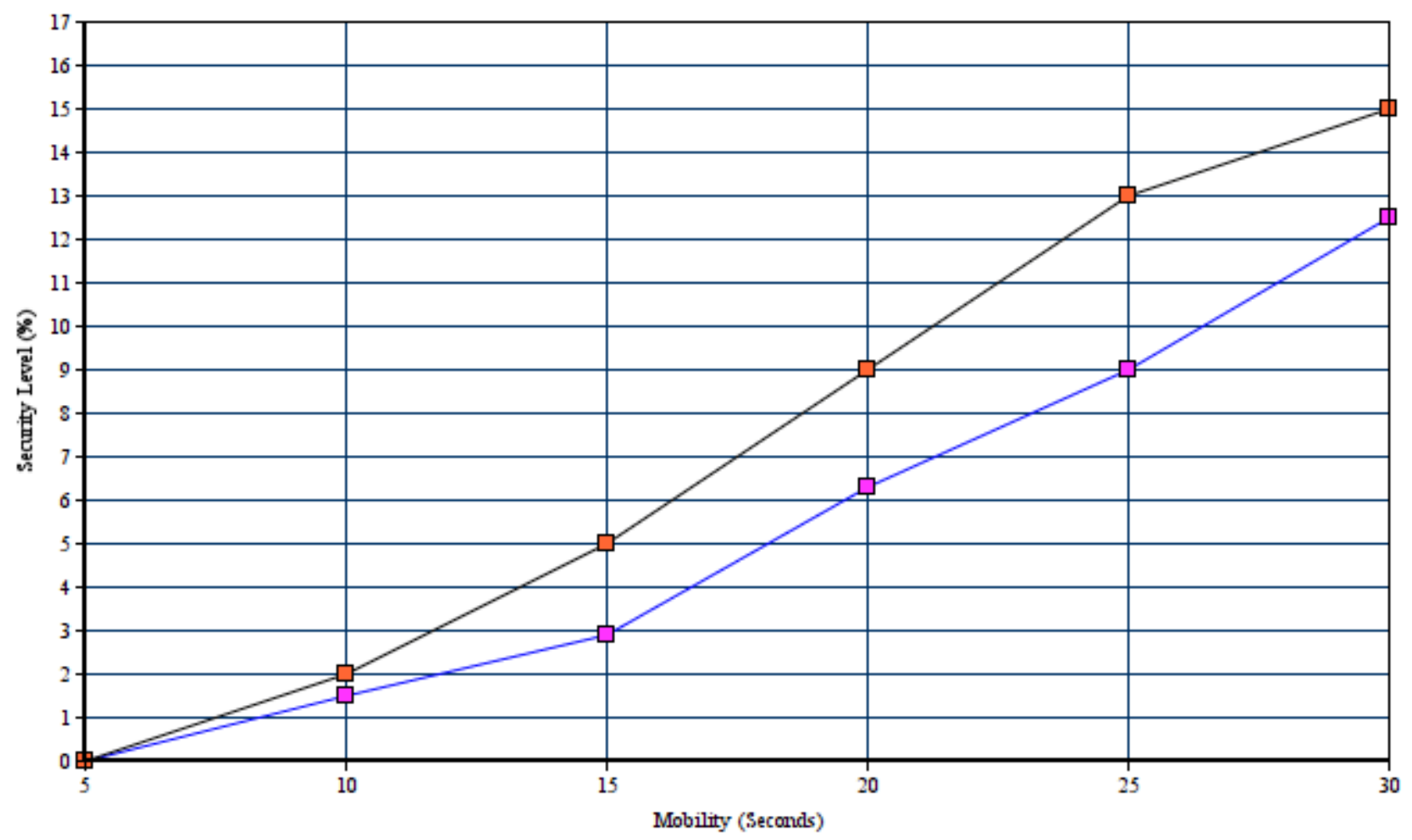

Figure 8

Security level with Mobility

๑- OMSR $\square$ LTRC-ORC

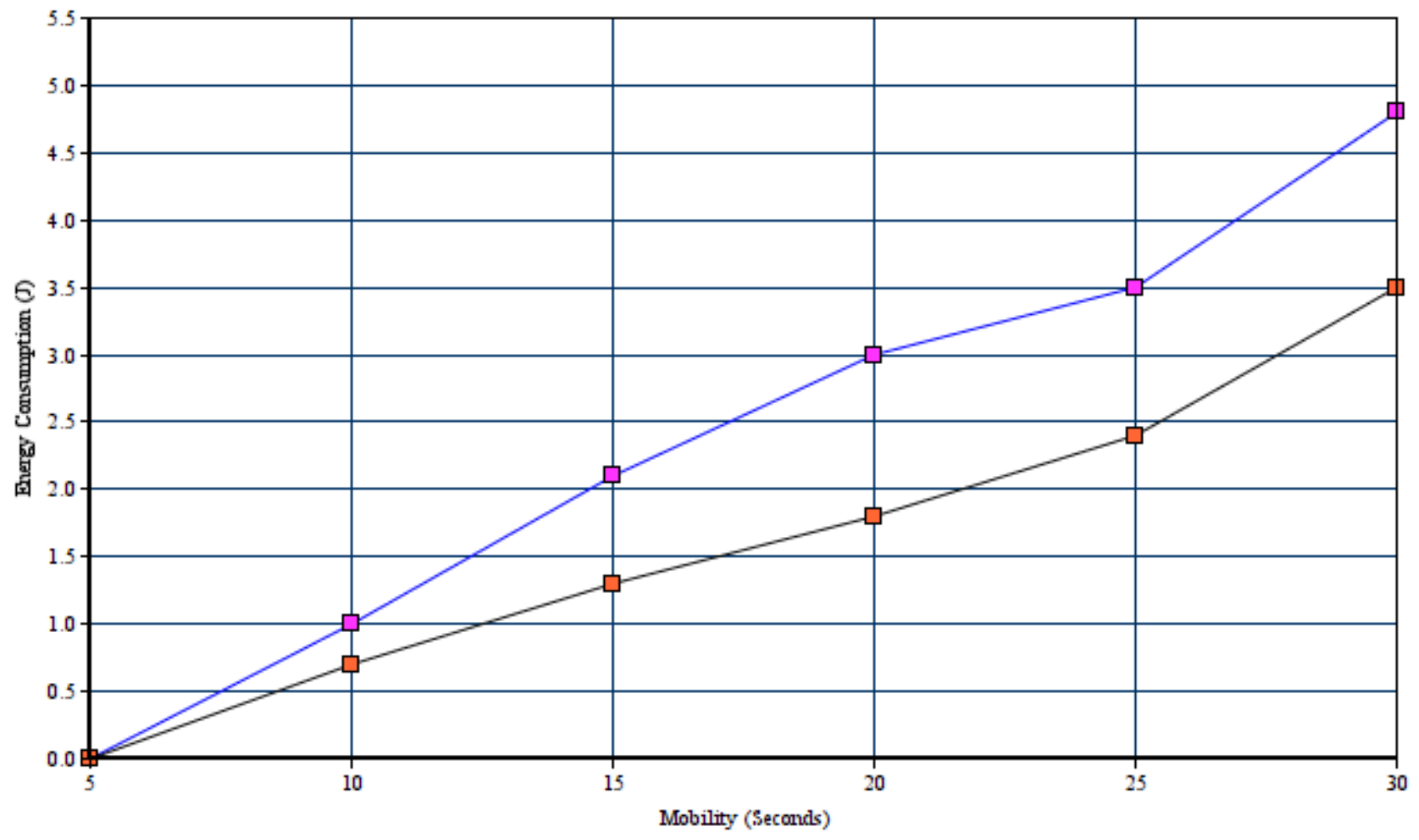


Figure 9

Energy Consumption with Mobility - $\square$ OMSR $\square$ - LTRC-ORC

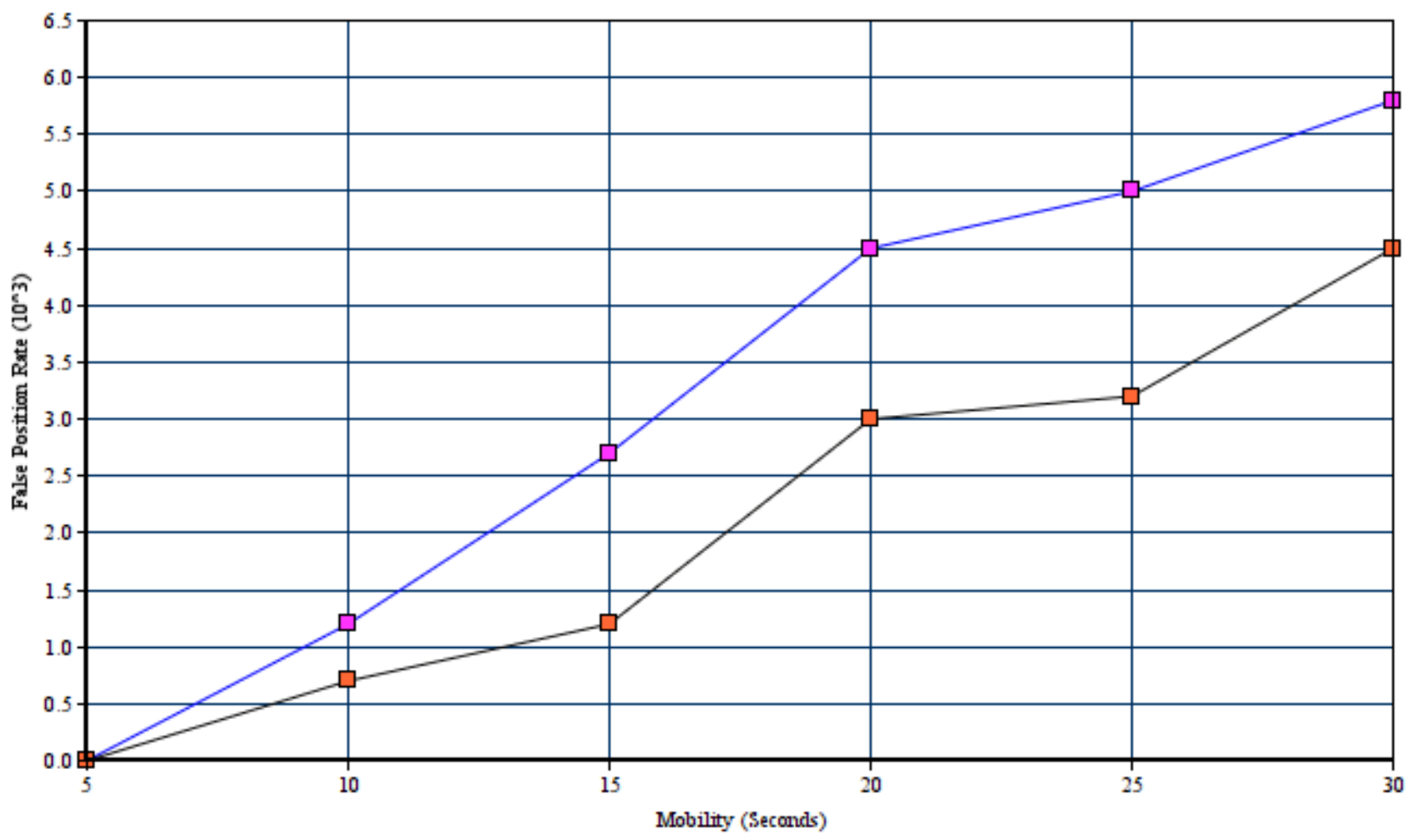

Figure 10

False Position Rate with Mobility 


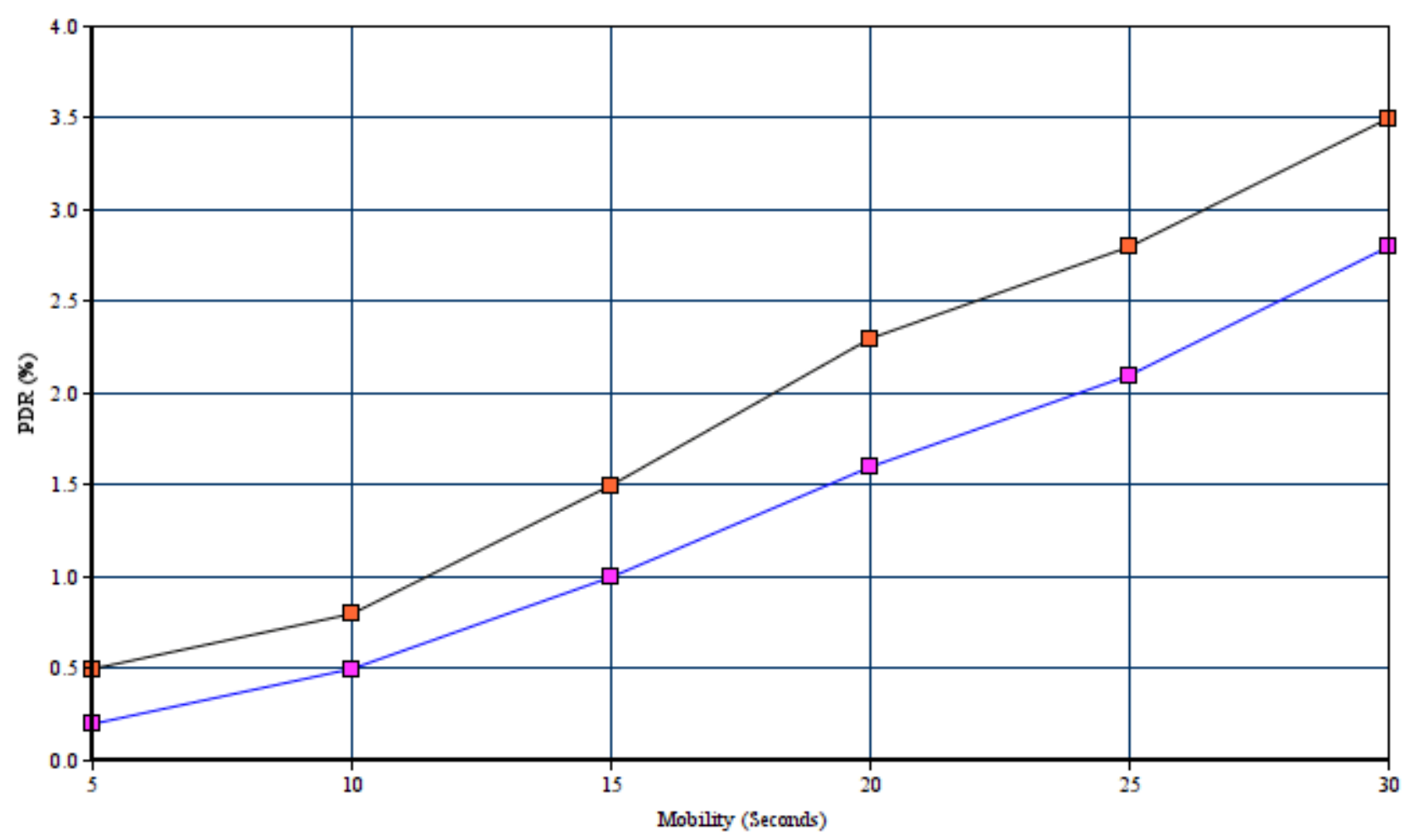

Figure 11

Packet Delivery Ration with Mobility

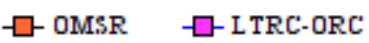

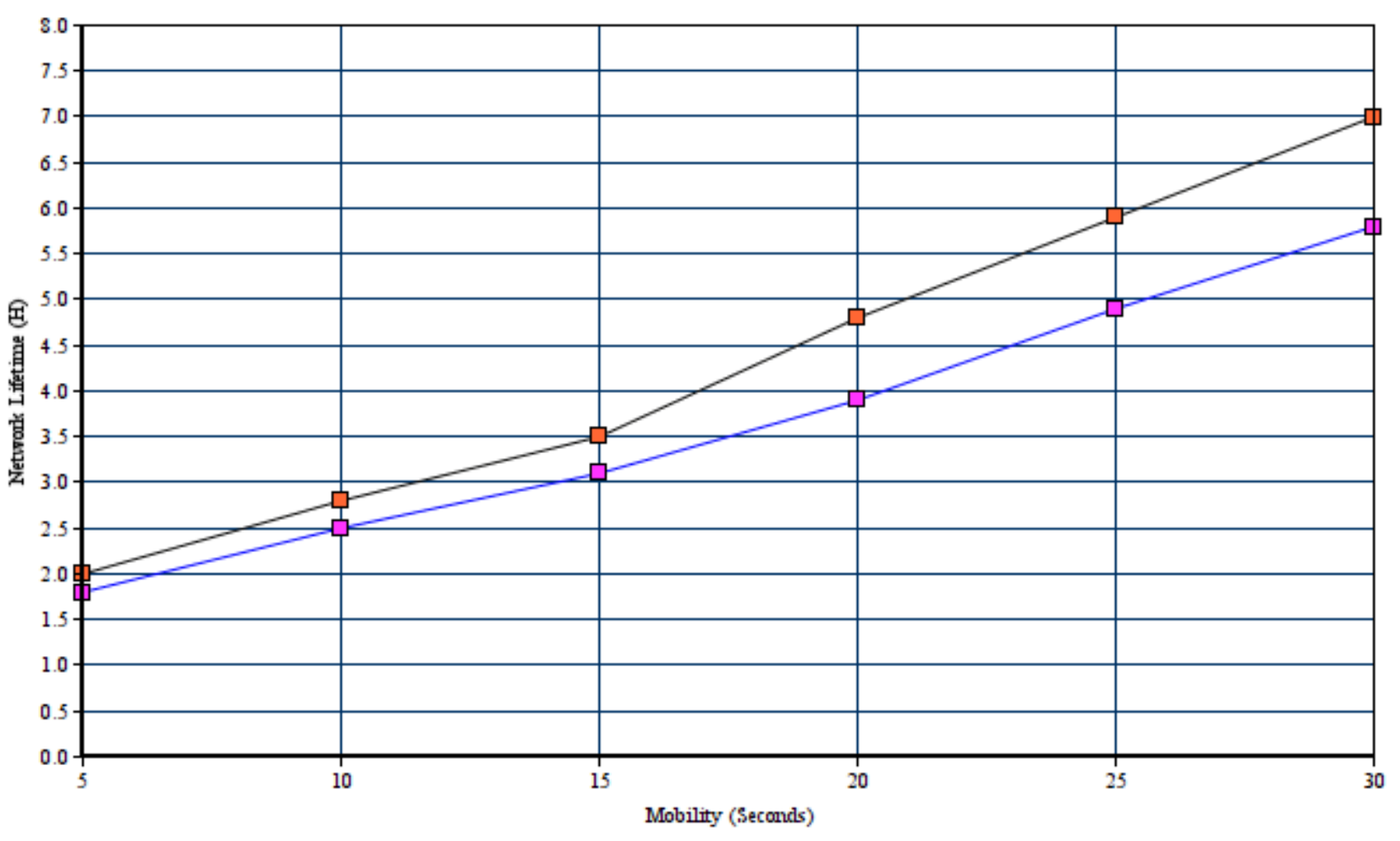

Figure 12 
Network Lifetime with Mobility

$-\square$ OMSR $\square-$ ITRC-ORC

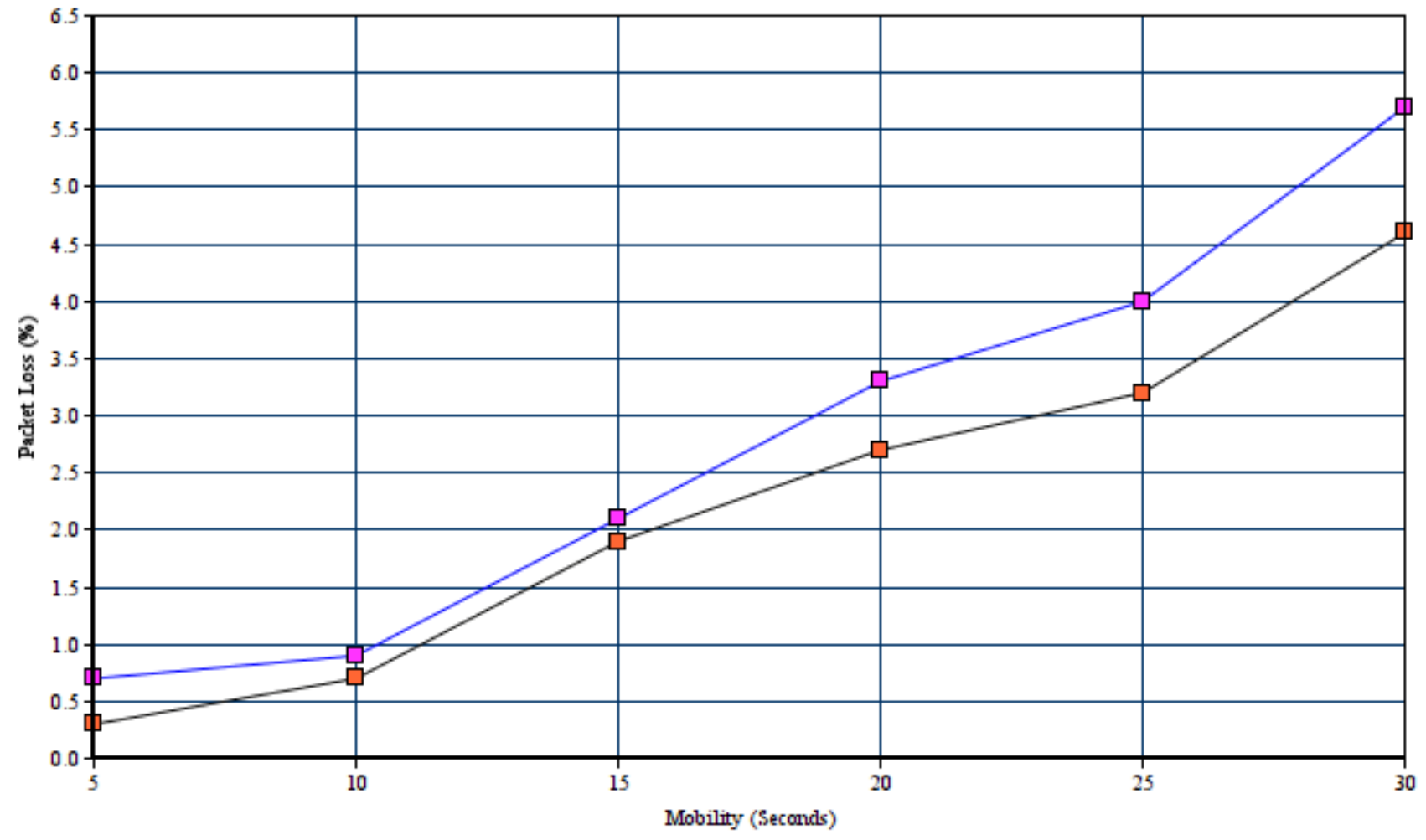

Figure 13

Packet Loss with Mobility 